\title{
Lactobacillus paracasei DSMZ16671 Reduces Mutans Streptococci: A Short-Term Pilot Study
}

\author{
Caterina Holz $\cdot$ Christiane Alexander $\cdot$ Christina Balcke $\cdot$ Margret Moré • \\ Annegret Auinger • Maren Bauer • Lauren Junker · Jörg Grünwald • \\ Christine Lang • Markus Pompejus
}

Published online: 8 September 2013

(c) The Author(s) 2013. This article is published with open access at Springerlink.com

\begin{abstract}
Reducing the burden of pathogenic mutans streptococci is a goal of oral health. Lactobacillus paracasei DSMZ16671, even after heat-killing, specifically coaggregates mutans streptococci in vitro and retains this activity in human saliva. In rats, it reduces mutans streptococcal colonization of teeth and caries scores. This pilot study sought to assess the potential of heat-killed $L$. paracasei DSMZ16671 (pro-t-action $^{\circledR}$ ) to reduce levels of salivary mutans streptococci in humans, using sugar-free candies as a delivery vehicle. A randomized, placebocontrolled, double-blind in vivo study of three groups examined the short-term effect of sugar-free candies containing 0 (placebo), 1 , or $2 \mathrm{mg} /$ candy piece of heat-killed
\end{abstract}

C. Holz $\cdot$ C. Balcke $\cdot$ C. Lang $(\bowtie)$

ORGANOBALANCE GmbH, Gustav-Meyer-Allee 25,

13355 Berlin, Germany

e-mail: lang@organobalance.de

C. Alexander · M. Moré · A. Auinger · J. Grünwald analyze\&realize AG, Waldseeweg 6, 13467 Berlin, Germany

M. Bauer · M. Pompejus

BASF New Business GmbH, 4 Gartenweg-Z25,

67063 Ludwigshafen, Germany

Present Address:

M. Bauer

BASF SE, Carl-Bosch-Strasse 38, 67063 Ludwigshafen,

Germany

L. Junker

BASF Corporation, Fine Chemical and Biocatalysis Research, 500 White Plains Road, Tarrytown, NY 10591, USA

Present Address:

M. Pompejus

BASF Corporation, Research Bioactive Materials,

500 White Plains Road, Tarrytown, NY 10591, USA
L. paracasei DSMZ16671 on the levels of salivary mutans streptococci determined before and after consumption of the candies. The candies were consumed 4 times during 1.5 consecutive days. Compared to the placebo group, the test groups' saliva had significantly reduced mutans streptococci as an immediate effect. These results suggest the use of heat-killed L. paracasei DSMZ16671 in suckable candies as a method to reduce mutans streptococci in the mouth and, thereby, caries risk. We think this a new concept and strategy for caries prevention and management.

Keywords Dental caries - Humans ·

Sugar-free candy $\cdot$ Lactobacillus pro-t-action .

Co-aggregation $\cdot$ Saliva

\section{Introduction}

Global increase in the prevalence of dental caries is a significant health problem, and reduced colonization of mutans streptococci is correlated with reduced risk of caries occurrence [1-3]. While there is some indication that caries is a multi-microbial disease [4], mutans streptococci are considered the predominant caries inducing microbial group with special importance in early colonization in children, and caries is associated with increased prevalence of mutans streptococci in the dental plaque biofilm adherent to teeth [5-7].

Mutans streptococci use teeth as primary colonization sites [8]. These bacteria are shed into saliva during oral function. From suspension in saliva, mutans streptococci can either re-adhere to the teeth or existing plaque biofilm or be swallowed. Prevention of their re-adhesion to the plaque by specific co-aggregation with $L$. paracasei DSMZ16671, promoting their oral clearance, could thus 
provide a strategy to reduce a key caries risk factor, benefiting oral health without generalized non-specific oral floral disruption [9]. The Lactobacillus cells co-aggregate with the mutans streptococci that are in suspension in the saliva.

Lactobacillus paracasei DSMZ16671 selectively coaggregates with mutans streptococci in vitro and in saliva, but does not co-aggregate other commensal bacteria of the oral microflora [10]. In rats, L. paracasei DSMZ16671 inhibits $S$. mutans colonization of teeth and significantly reduces caries in rats already infected by it [9].

As a test of the hypothesis that this strategy for caries prevention can be applied to humans, we conducted a short-term, pilot, in vivo, randomized, placebo-controlled, double-blind study using sugar-free candies as the heatkilled L. paracasei DSMZ16671 delivery vehicle.

\section{Materials and Methods}

\section{Test Products}

Boiled, hard, sugar-free candies that were either supplemented or not supplemented with L. paracasei DSMZ16671 were produced for this study by DR.C SOLDAN ${ }^{\circledR} \mathrm{GmbH}$ (Nuremberg, Germany), a company certified to have met the requirements of International Food Standard (IFS) version 5 for the development, manufacture, and packaging of drops, fruit jelly sweets, and pastilles (category 14). The pasteurized and spray-dried L. paracasei DSMZ16671 had been added as the active ingredient in its commercially available form (Lactobacillus pro-t-action ${ }^{\circledR}$, BASF Future Business GmbH, Ludwigshafen, Germany). The dosage of L. paracasei DSMZ16671 in the candies was confirmed from the sodium content of the candies as measured by atomic spectrometry. Briefly, the candies were produced by mixing, then boiling, all ingredients (except flavoring agents and $L$. paracasei DSMZ16671) until achieving a homogeneous melt. The melt was then cooled to $80{ }^{\circ} \mathrm{C}$, whereupon flavor and $L$. paracasei DSMZ16671 were added, while mixing. This hot melt was pressed into forms, cooled, and packaged as candies. Basic ingredients for the test candies were isomaltitol, maltitol, water, sucralose, and mint oil. Test and placebo candies were identical in weight $(2.5 \mathrm{~g})$, size, color, and flavor.

\section{Study Design and Subjects}

A randomized, placebo-controlled, double-blind, in vivo study was done with 3 study arms: placebo, test group 1 (1 mg L. paracasei DSMZ16671/candy), and test group 2 (2 mg L. paracasei DSMZ16671/candy). Study subjects $(n=60)$ gave informed written consent consistent with
ICH Good Clinical Practice guidelines. This study was conducted at a single center in Berlin, Germany. As the trial was not a clinical trial, an ethical committee did not need to be considered, and the trial was not registered, as at the time of the trial in Germany, it was not customary for pilot-type trials to be registered.

This clinical trial was designed as a pilot to gain information regarding the treatment effect. Although there was not enough data for a sample size calculation, the trial proceeded with a sample size of 20 subjects per study group. The randomization procedure was a block randomization of 10 blocks with a block size of 6 . Randomization was performed beforehand and the resulting allocation was recorded in the allocation lists. The participants those administering the treatment and those evaluating the results were blinded to the group assignment.

Subjects were excluded if under current treatment for oral or pharyngeal inflammation, restorative, gingival/ periodontal, or oral surgical problems, brushed their teeth more than three times daily, used antibiotics or had dental hygiene treatment in the past 6 weeks, used full artificial dentures, were currently pregnant or lactating, had history of alcohol abuse or drug addiction, or had German language difficulties. Inclusion criteria were good health, age over 18, and written consent. There was no preselection screening of subjects for baseline levels of mutans streptococci.

\section{Test Schedule}

The study was conducted according to the protocol and was performed over two successive days. Subjects were not to perform any oral hygiene activities or consume coffee, tea, wine, probiotic foods (such as yoghurt or cheese) from the evening prior to the first study day until completion of the study 1.5 days later. They were instructed to expectorate one $\mathrm{mL}$ of saliva $(\mathrm{R}-1)$ into a sterile $15-\mathrm{mL}$ polyethylene (PE) tube, without any mechanical or taste stimulation, upon rising. This sample (R1) was to be transported to the study site within $1 \mathrm{~h}$. At the study site, a second saliva sample (Pre-1) was collected before candy use. All subjects were then randomized to one of three treatment groups (placebo, test 1, or test 2) and were instructed to consume a single assigned candy piece by sucking, until fully consumed, and to refrain from chewing. The assigned candies were provided in packages coded so as to keep subjects and investigators blind. Immediately (no later than $10 \mathrm{~min}$ ) after consuming the candy, the subjects collected another saliva sample (Post-1). Subjects who had removable partial dentures wore them throughout all study procedures.

Each subject consumed 3 more candies, one at a time, during the day, 10 min after each unsupervised meal, and 
was instructed not to exchange candies with other study participants.

On day 2, all subjects again collected a saliva sample upon rising (R-2), and another saliva sample (Pre-2) was collected at the study site before sucking another identically coded candy. A final saliva sample (Post-2) was collected within $10 \mathrm{~min}$ after consumption of this candy.

\section{Sampling and Sample Analysis}

Except for the unsupervised saliva samples collected early in the mornings upon rising, all samples were collected onsite, under supervision by trained clinical staff. The samples were kept at room temperature and transported to the microbiology facility, and processed within a maximum of $2 \mathrm{~h}$ of collection, which had been established in pretests to give reliable and reproducible results (CFU). They were homogenized by sonication (15 min, ultrasonic bath Bandelin Sonorex GT120), vortexed, and a $0.5 \mathrm{~mL}$ volume of each was transferred into a sterile PE tube, diluted with $4.5 \mathrm{~mL}$ phosphate-buffered saline, and re-vortexed. One milliliter aliquot of 1:100 and 1:500 dilutions was spread in duplicate onto selective TSY20B agar [11]. The TSYB medium which was used for the detection and quantification is selective for mutans streptococci (S. mutans, S. sobrinus, etc.). The medium contains Bacitracin, which is an antibiotic selective for mutans streptococci among other bacteria. The plates were incubated anaerobically at $37{ }^{\circ} \mathrm{C}$ for 5 days and $\mathrm{CFU} / \mathrm{mL}$ of saliva determined. Colonies were confirmed as $S$. mutans by random PCR checks using specific primers (Sm F5: AGCCATGCGCAATCAACAGGTT; Sm R4: CGCAACGCGAACATCTTGATCAG [12]). Data were recorded on Case Report Forms.

\section{Primary Outcome Measures}

The primary outcome measure was the group change in salivary concentration of mutans streptococci immediately before (Pre-1, day 1 and Pre-2, day 2) and after (Post-1, day 1 and Post-2, day 2) consuming the test candies as compared to placebo candy. A second outcome measure was the individual study subject change in salivary mutans concentration.

\section{Product Acceptability Evaluation}

Study subjects were asked to rate the acceptability of candies through an interview conducted by the blinded investigator.

\section{Statistical Methods}

As mutans streptococcal counts were not normally distributed, as expected [5], all values were log-transformed to improve distribution for parametric analysis. Per protocol, data sets with less than $1,500 \mathrm{CFU} / \mathrm{mL}$ were excluded from further consideration due to the limit of detection.

Frequency distributions were analyzed using the chisquare test $\left(p_{\text {chi-value }}\right)$. The Kruskal-Wallis $\left(p_{\mathrm{KW}}\right.$-value) method was employed to test for among-group comparisons (placebo vs. test groups). Comparisons within a group (e.g., pre- vs. post-consumption) were performed using the Wilcoxon signed-rank test $\left(p_{\mathrm{Wil}}\right)$. The mean relative difference in the number of mutans streptococci before (Pre1) and after (Post-1) candy consumption was calculated for all three groups, for day one [Post-1 vs. Pre-1] and day two [Post-2 vs. Pre-2]. Statistical analyses were performed with SPSS (SPSS for Windows, Release 19, LEAD Technologies, Inc.). Values of $p<0.05$ were considered significant. Means were given with $95 \%$ confidence intervals. Nonparametric analyses were performed because of the small sample size and its emphasis on the qualitative results. The $t$ test was used for quantitative descriptions. CI was presented for this pilot study and represents the individual range of data and provides information about the variance of the data.

\section{Results}

All 60 subjects were enrolled, randomized, and completed the trial. There were no statistically significant $(p>0.05)$ differences in the baseline characteristics among the sixty (60) subjects after random assignment to groups for age, gender, height, weight, BMI, heart rate, blood pressure, number of teeth, or number of smokers or vegetarians. All subjects were Caucasian. None had oral inflammatory lesions.

\section{Safety and Product Acceptability}

No adverse events occurred in any group. For all groups, candy acceptability was rated good or very good $\left(p_{\text {chi }}>0.05\right)$.

Range of Level of Mutans Streptococci in Baseline Samples

The majority ( $86.7 \%$ on day 1 and $86.5 \%$ on day 2 ) of the mutans streptococcal counts were between $10^{3}$ and $10^{5}$ CFU/mL saliva (Table 1). Counts exceeding $10^{6} \mathrm{CFU} / \mathrm{mL}$ were observed infrequently (13.3\% on day 1 and $13.5 \%$ on day 2). Very low levels of less than $10^{4} / \mathrm{mL}$ were observed in 8 out of 60 test persons $(13.3 \%)$. There was no statistically significant difference between the study groups at baseline samples collected at R-1, R-2, Pre-1, and Pre-2. The ranges of mutans streptococcal levels in the samples were comparable in all groups. R-1 values-values given 
Table 1 Log-transformed means and $95 \%$ confidence intervals of mutans streptococci in samples taken upon rising $(R)$, before (pre) and after (post) consuming one candy, either containing $1 \mathrm{mg}$ or $2 \mathrm{mg}$ of L. paracasei DSMZ16671 or placebo

\begin{tabular}{|c|c|c|c|c|c|}
\hline & Upon rising $(R)$ & $\begin{array}{l}\text { Pre-consumption } \\
\text { (pre) }\end{array}$ & $\begin{array}{l}\text { Post-consumption } \\
\text { (post) }\end{array}$ & $\begin{array}{l}\text { Absolute change } \\
\text { (pre-post) }\end{array}$ & $p_{\mathrm{Wil}}^{\#}$ \\
\hline \multicolumn{6}{|l|}{ Day 1} \\
\hline Placebo $(n=19)$ & $5.16(4.78 ; 5.55)$ & $5.02(4.63 ; 5.40)$ & $4.81(4.38 ; 5.24)$ & $0.21(0.00 ; 0.41)$ & 0.053 \\
\hline Test group $1(n=17)(1 \mathrm{mg}$ DSMZ16671) & $5.36(4.92 ; 5.80)$ & $5.23(4.82 ; 5.64)$ & $5.11(4.73 ; 5.49)$ & $0.12(-0.02 ; 0.26)$ & 0.084 \\
\hline Test group $2(n=20)(2 \mathrm{mg}$ DSMZ16671) & $5.23(4.87 ; 5.59)$ & $5.16(4.84 ; 5.47)$ & $4.89(4.52 ; 5.25)$ & $0.27(0.14 ; 0.41)$ & 0.001 \\
\hline \multicolumn{6}{|l|}{ Day 2} \\
\hline Placebo $(n=17)$ & $5.11(4.62 ; 5.61)$ & $4.94(4.46 ; 5.43)$ & $4.86(4.36 ; 5.36)$ & $0.08(-0.13 ; 0.30)$ & 0.215 \\
\hline Test group $1(n=12)(1 \mathrm{mg}$ DSMZ16671) & $5.54(5,21 ; 5,86)$ & $5.43(5.09 ; 5.77)$ & $5.27(4.93 ; 5.61)$ & $0.16(0.04 ; 0.28)$ & 0.009 \\
\hline Test group $2(n=15)(2 \mathrm{mg}$ DSMZ16671) & $5.47(5.10 ; 5.83)$ & $5.43(4.98 ; 5.71)$ & $5.10(4.73 ; 5.47)$ & $0.25(0.12 ; 0.37)$ & 0.002 \\
\hline
\end{tabular}

\# $p$ values (Wilcoxon-rank sum test) for changes from baseline (pre- vs. post-consumption)

are log-transformed data-were $4.92 \pm 0.89$ for placebo, $4.95 \pm 1.01$ for test group 1, and $5.16 \pm 0.88$ for test group 2 ( $\left.p_{\mathrm{KW}} 0.720\right)$.

The samples taken on-site before consuming the candies (Pre-1 and Pre-2) were used as baseline values for the evaluation of the immediate effect of the candies on salivary level of mutans streptococci.

\section{Immediate Reduction in the Level of Mutans Streptococci After Candy Consumption}

Table 1 shows that post-consumption salivary level of mutans streptococci compared to pre-consumption was significantly reduced in test group $2(2 \mathrm{mg} /$ piece $)$ both on day one $\left(p_{\mathrm{Wil}}=0.001\right)$ and on day $2\left(p_{\mathrm{Wil}}=0.002\right)$. On day 2 (after day 1 consumption of 4 candies), also test group 1 (1 mg/ piece) had lower salivary mutans recoveries after candy consumption compared to the pre-consumption level $\left(p_{\text {Wil }}=0.009\right)$. The level of mutans streptococci did not differ significantly in the placebo group on the 2 days of the study.

In view of the high variability in baseline counts among subjects, effects of treatments on relative changes in salivary mutans streptococcal concentration were also evaluated on a per-subject basis. On day 1, after intake of one candy, $75 \%$ of the subjects taking placebo (ns), $65 \%$ in test group 1 (ns), and $85 \%$ test group $2\left(p_{\text {Wil }}=0.003\right)$ had reduced level of mutans streptococci. On day 2, after intake of 5 candies (four on day 1 , one on day 2), only $59 \%$ of the subjects taking placebo candies had decreased salivary mutans streptococcal counts (ns), whereas $80 \%$ of test group 1 subjects and $75 \%$ (both, $p_{\mathrm{Wil}}=0.005$ ) of test group 2 subjects had reduced salivary mutans streptococcal counts.

\section{Discussion}

The results presented here as a pilot study in human adults evidence that the use of $L$. paracasei DSMZ16671, contained in an acceptable food commonly consumed, lowers salivary levels of Strep. mutans. This reduction is significant on two consecutive days for the higher concentration consumed and it is seen as a trend for the lower concentration candies. The data are consistent with those of Lang et al. [10] that in vitro, even when heat-killed and incubated with saliva, these bacteria cause specific coaggregation with mutans streptococci in planktonic suspension and with those of Tanzer et al. [9] that these heatkilled bacteria when eaten with the highly cariogenic diet of rats already colonized by mutans streptococci reduce their levels on the teeth, reduce caries experience, and appear to foster clearance from the mouth. Thus, the present pilot human data support our understanding that these bacteria, even when heat-killed and contained in a sugar-free candy vehicle, foster specific co-aggregation with mutans streptococci in planktonic suspension in saliva and, thereby, an increased probability that they will be cleared from the mouth. We think it remarkable that this effect was observed after exposure to only five pieces of candy containing 1 or $2 \mathrm{mg}$ of dead L. paracasei DSMZ16671 consumed in 1.5 days.

This double-blind, randomized, placebo-controlled in vivo study demonstrated immediate short-term reductions on salivary mutans streptococci levels through the consumption of L. paracasei DSMZ16671 in candies. Test subjects were not preselected according to their mutans streptococcal levels in the oral cavity. While this represented "real-life conditions," it did probably contribute to statistical variation. Future studies are planned with larger populations with more homogeneous mutans streptococcal level subjects.

Incorporation of L. paracasei DSMZ16671 into sugarfree candies is but one possible form of application of prot-action ${ }^{\circledR}$. Additionally, sugar-free candies stimulate saliva flow, a benefit to oral health $[13,14]$. We could not observe any significant decrease in mutans streptococcal counts by saliva flow per se. Rather, the slight, albeit non-statistically 
significant reduction of salivary mutans streptococci in the placebo-candy-using group is probably attributable to the Hawthorne effect in which the mere participation in a study can change study participant behavior if subjects believe the investigators are interested in a particular outcome of such behavior, familiar in the dental clinical research literature [15-17].

Release of L. paracasei DSMZ16671 into saliva from sugar-free candies, leading to co-aggregation with mutans streptococci, with resultant increased probability of swallowing rather than re-attachment to the teeth or plaque biofilm $[9,10]$ is a plausible explanation for the reduction in the prime-albeit not sole [4]—caries pathogen in humans' saliva.

Heat-killed L. paracasei DSMZ16671 has the potential to offer a method for selective reduction in mutans streptococci, thereby positively influencing the oral microbiota, without adding new or additional risk factors, as might be the case with the addition of live lactobacilli, especially those without ability to specifically co-aggregate with mutans streptococci.

Acknowledgments This research was funded by BASF New Business GmbH. The authors thank Norman Bitterlich for his expert support with statistical analysis.

Conflict of interest Caterina Holz, Christiane Alexander, Christina Balcke, Margret Moré, Annegret Auinger, Maren Bauer, Lauren Junker, Jörg Grünwald, Christine Lang, and Markus Pompejus declare that they have no conflict of interests.

Ethical standards All procedures followed were in accordance with the ethical standards of the responsible committee on human experimentation (institutional and national) and with the Declaration of Helsinki 1975, as revised in 2008. Informed consent was obtained from all patients included in the study.

Open Access This article is distributed under the terms of the Creative Commons Attribution License which permits any use, distribution, and reproduction in any medium, provided the original author(s) and the source are credited.

\section{References}

1. Bagramian RA, Garcia-Godoy F, Volpe AR (2009) The global increase in dental caries. A pending public health crisis. Am J Dent 22:3-8
2. U.S. Department of Health and Human Services. National Call to Action to Promote Oral Health. U.S. Department of Health and Human Services, Public Health Service, National Institutes of Health, National Institute of Dental and Craniofacial Research, Rockville. NIH Publication No. 03-5303, Spring 2003

3. Alamoudi NM, Hanno AG, Masoud MI, Sabbagh HJ, Almushayt AS, Masoud IM (2012) Effects of xylitol on salivary mutans streptococcus, plaque level, and caries activity in a group of Saudi mother-child pairs. An 18-month clinical trial. Saudi Med J 33:186-192

4. Alcaraz LD, Belda-Ferre P, Cabrera-Rubio R, Romero H, SimónSoro A, Pignatelli M, Mira A (2012) Identifying a healthy oral microbiome through metagenomics. Clin Microbiol Infect 18(Suppl 4):54-57

5. Loesche WJ (1986) Role of Streptococcus mutans in human dental decay. Microbiol Rev 50:353-380

6. Tanzer JM, Livingston J, Thompson AM (2001) The microbiology of primary dental caries in humans. J Dent Educ 65:1028-1037

7. Islam B, Khan SN, Khan AU (2007) Dental caries: from infection to prevention. Med Sci Monit 13:RA196-203

8. Gibbons RJ, Cohen L, Hay DI (1986) Strains of Streptococcus mutans and Streptococcus sobrinus attach to different pellicle receptors. Infect Immun 52:555-561

9. Tanzer JM, Thompson A, Lang C, Hareng L, Garner A, Reindl A, Pompejus M (2010) Caries inhibition by and safety of Lactobacillus paracasei DSMZ16671. J Dent Res 89:921-926

10. Lang C, Böttner M, Holz C, Veen M, Ryser M, Reindl A, Pompejus M, Tanzer JM (2010) Specific Lactobacillus/mutans streptococcus co-aggregation. J Dent Res 89:175-179

11. Schaeken MJMM, van der Hoeven JS, Franken HCM (1986) Comparative recovery of Streptococcus mutans on five isolation media, including a new simple selective medium. J Dent Res 65:906-908

12. Yano A, Kaneko N, Ida H, Yamaguchi T, Hanada N (2002) Realtime PCR for quantification of Streptococcus mutans. FEMS Microbiol Lett 214:23-30

13. Edgar WM (1998) Sugar substitutes, chewing gum and dental caries: a review. Br Dent J 184:29-32

14. Dodds MW, Johnson DA, Yeh CK (2005) Health benefits of saliva: a review. J Dent 33:223-233

15. Owens J, Addy M, Faulkner J (1997) An 18-week home-use study comparing the oral hygiene and gingival health benefits of triclosan and fluoride toothpastes. J Clin Periodontol 24:626-631

16. De Amici D, Klersy C, Ramajoli F, Brustia L, Politi P (2000) Impact of the Hawthorne effect in a longitudinal clinical study: the case of anesthesia. Control Clin Trials 21:103-114

17. Feil PH, Grauer JS, Gadbury-Amyot CC, Kula K, McCunniff MD (2002) Intentional use of the Hawthorne effect to improve oral hygiene compliance in orthodontic patients. J Dent Educ 66: $1129-1135$ 